\title{
Fluorescence Decay Heterogeneity Model Based on Electron Transfer Processes in an Enzyme-Ligand Complex
}

\author{
J. WLODARCZYK AND B. KIERDASZUK* \\ University of Warsaw, Institute of Experimental Physics \\ Department of Biophysics, Żwirki i Wigury 93, 02-089 Warsaw, Poland
}

(Received December 28, 2004; in final form February 7, 2005)

The models are described for complex fluorescence decay of tyrosine in proteins involving continuous distribution of fluorescence lifetimes and electron transfer processes. We introduce the analytical decay function with a power-like term, which provides good fits to highly complex fluorescence decays. Moreover, the power-like term in the proposed decay functions is a manifestation of so-called Tsallis nonextensive statistics and is suitable for description of the systems with long-range interactions, memory effect, as well as with fluctuations of the characteristic lifetime of fluorescence. The proposed decay functions were applied to analysis of fluorescence decays of tyrosine in a protein, i.e. the enzyme purine nucleoside phosphorylase from E. coli, free in aqueous solution and in the complex with formycin A (an inhibitor) and orthophosphate (a co-substrate), and demonstrated that both models reflect the enzyme-ligand interactions. Direct measure of heterogeneity of the enzyme systems is provided by a variance of fluorescence lifetime distribution. The possible number of deactivation channels and excited state mean lifetime can be easily derived without a priori knowledge of the complexity of studied system.

PACS numbers: 33.50.-j

\section{Introduction}

There are many natural physical processes which follow a power-like distribution. The power law behavior is commonly observed in a variety of systems

*corresponding author; e-mail: borys@biogeo.uw.edu.pl 
ranging from cosmology, fluids, to econophysics and social science, among others $[1,2]$. In particular, complex systems characterized by long-range correlations, long-range microscopic memories, or fluctuations of some parameters present in the system, display a non-exponential behavior. They can be described in terms of nonextensive statistics introduced in 1988 by Tsallis [3]. In this approach a power-like formula (one parameter $q$-exponential function)

$$
e_{q}^{t} \equiv\left[1-(1-q) \frac{t}{\tau}\right]^{\frac{1}{1-q}} \stackrel{q \rightarrow 1}{\longrightarrow} e_{q=1}^{t} \equiv \exp \left(-\frac{t}{\tau}\right)
$$

is a generalization of the Boltzmann-Gibbs function, replaces the usual exponential function, and reproduces it for $q \rightarrow 1$.

Biological systems, particularly biomolecules (e.g. proteins and their complexes with ligands) can be treated as good examples of complex systems, which exist in a large variety of energetic states corresponding to different conformations [4]. Therefore proteins are ideal samples to study relaxation in complex systems such as alloys of the disordered energetic states.

In this paper, addressing the decay of singlet excited states of proteins, we display ways of modeling such systems, leading to a long-time power-like behavior. We show that the underlying reason for non-exponential decay of protein fluorescence is a distribution of a characteristic lifetime of the fluorophore and excitation transport processes. The latter was previously applied in studies of excitation transport in solids [5]. In this paper, we propose a model based on the electron transport and show its applicability to description of fluorescence decays in protein systems.

We start with a brief description of our present knowledge, relevant to interpretation of non-exponential relaxation of biological molecules.

\subsection{Previous models}

Decays of fluorescence intensity of proteins often exhibit a non-exponential behavior, the origin of which is usually interpreted in terms of multiple (ground state) conformations (i.e. rotamer model) or excited-state processes (i.e. dipolar relaxations) and described by a multi-exponential function [6]. The rotamer model is based on the interpretation of each discrete exponential component with the aid of a particular protein conformation, including dynamic equilibrium between rotational isomers of tryptophan [7] and tyrosine $[8,9]$ residues. However, there are many difficulties with the application of this model, e.g. X-ray or NMR data do not indicate multiple occupancy of rotameric states [10]. There are many proteins for which it is difficult to build a molecular model with the alternative orientations of fluorophores. Also the case of multitude conformational substates in proteins with possible time-dependent interconversions between them [11], which are not slow in comparison with fluorescence lifetimes, cannot be properly described by this model. Even for the indole moiety in solution, it is difficult to find unique conformations corresponding to discrete fluorescence decay components $[12,13]$. 
Also studies on the ${ }^{1} L_{a}$ and ${ }^{1} L_{b}$ transitions of tryptophan do not lead to an explanation of protein fluorescence heterogeneity $[14,15]$.

The second model mentioned above involves the excited state processes and assumes that the dipole moment associated with an excited fluorophore changes the direction and magnitude after electronic excitation. The mobile polar molecules in close proximity to the fluorophore reorientationally relax, and their relaxation occurs from many partially relaxed states. The relaxation processes affect the kinetic of the decay and lead to a decay in a non-exponential form (i.e. not properly described by a sum of exponential terms). All the foregoing suggest that continuous lifetime distributions are more relevant models than a sum of discrete exponential terms. There are many other cases, where distribution of decay times is expected rather than the limited number of discrete decay times [16], e.g. a fluorophore in a mixture of solvents, a case of co-existence of the range of environments in solution, or in proteins with many fluorophore residues. In such cases we should consider a more adequate model to describe complex fluorescence relaxation linking the character of the decay law to the distribution of lifetimes and/or excitation transfer process.

\subsection{Lifetime distribution}

Previous investigations indicate that a detailed analysis of the energy level distribution in proteins is required for understanding the related relaxation process in these systems. It is known that proteins exist in different energy levels corresponding to different protein conformations, with allowance for transitions between them. For such a system, the effective Hamiltonian $H$ (describing the transition between the $i$-th and $j$-th energy states, with coupling between them) is the $M \times M\left(H_{i j}=H_{j i}\right)$ matrix, where $M$ is the number of states. The Hamiltonian contains excitation energies and nearest neighbor interactions, as well as interaction between transition dipoles. The interactions between transition dipoles produce additional perturbation terms, which affect $H$. Moreover each physical copy of the system is different, and, therefore, the Hamiltonian described above should be studied in a statistical manner. When fluctuations are significantly high so that it can be treated as a random component, properties of ensembles of Hamiltonians can be studied in terms of random matrix theory (RMT) [17]. In particular, there are analytical method in this approach which allow one to study such a random system. In the limit, where the matrix size becomes large, it can be shown that the spectral properties are independent of the distribution for individual matrix elements. Using the RMT approach, it has been shown that the distribution $P\left(C_{i}\right)$ of amplitude $C_{i}$ for eigenvectors of $H$ (in the limit of large $M$ ) has the following form [18]:

$$
P\left(C_{i}\right)=\frac{1}{\sqrt{2 \pi\left\langle C_{i}^{2}\right\rangle}} \exp \left(-\frac{C_{i}^{2}}{2\left\langle C_{i}^{2}\right\rangle}\right) .
$$


For our application we are interested in the distribution of the decay rate $\sigma_{i}=1 / \tau_{i}$, which is proportional to the probability $C_{i}^{2}$ of finding a single state $i$ in the composite state. Taking into account that $P(\sigma) \mathrm{d} \sigma=2 P\left(C_{i}\right)\left|\mathrm{d} C_{i}\right|$, for a complex system with $N$ decay paths affecting the excited-state lifetime, the distribution of rates $P(\sigma)$ is given in the form of the gamma distribution [18], with a mean value $\langle\sigma\rangle=N\left\langle\sigma_{i}\right\rangle=N /\left\langle\tau_{i}\right\rangle$. Because $P(\tau) \mathrm{d} \tau / \tau^{2}=P(\sigma) \mathrm{d} \sigma$ and the mean lifetime value $\tau_{0}=1 /\langle\sigma\rangle$, the corresponding distribution of lifetimes $P(\tau)$ one can write in the form

$$
P_{N}(\tau) \mathrm{d} \tau=\frac{1}{\Gamma\left(\frac{N}{2}\right)}\left(\frac{N \tau_{0}}{2}\right)\left(\frac{N \tau_{0}}{2 \tau}\right)^{\frac{N}{2}-1} \exp \left(-\frac{N \tau_{0}}{2 \tau}\right) \frac{\mathrm{d} \tau}{\tau^{2}}
$$

which may result from relaxation of the fluorophore and/or its environment.

$P(\tau)$ is a continuous distribution of characteristic time scales in the considered system. It is worth noting that the gamma distribution is also predicted by a maximum entropy method as the most expected distribution under certain constrains [19]. Hence, taking into account the relaxation from many contributing components, the fluorescence decay $I(t)$ is determined by

$$
I(t)=I_{0} \int_{0}^{\infty} P_{N}(\tau) \exp \left(-\frac{t}{\tau}\right) \mathrm{d} \tau
$$

with normalization $I_{0}=I(t=0)$.

This leads directly to the decay function (see Refs. $[18,20]$ for details)

$$
I(t)=I_{0}\left[1-(1-q) \frac{t}{\tau_{0}}\right]^{\frac{1}{1-q}}
$$

described by the mean value of the lifetime distribution $\left(\tau_{0}\right)$ and one new parameter of heterogeneity $(q)$, defined as

$$
q=1+\frac{2}{N}=1+\frac{\left\langle(\sigma-\langle\sigma\rangle)^{2}\right\rangle}{\langle\sigma\rangle^{2}}
$$

and describing the relative variance (Eq. (6)) of fluctuations of $\sigma=1 / \tau$ around the $1 / \tau_{0}$ value. The mean decay time $\langle t\rangle$ is obtained directly from integration of Eq. (5), and is given by

$$
\langle t\rangle=\frac{\tau_{0}}{3-2 q} .
$$

Note that the power-like decay function is a manifestation of the Tsallis $q$-exponential function and results directly from fluctuations of the parameter $\sigma=1 / \tau$ in the exponential formula [20]. Moreover, Eq. (5) corresponds to a memory effect in the considered system. It stems from the rate equation $\mathrm{d} N(t)=-(1 / \tau(t)) N(t) \mathrm{d} t$ (describing probability of emission a photon at a given time $t$, by an excited residue at $t=0$ ) for linear time-dependence of the lifetime parameter [19] in the form $\tau(t)=\left[\tau_{0}-(1-q) t\right] / q$. 
Since protein structural fluctuations occur in the nanosecond-picosecond time scale [21, 22], these rapid fluctuations would affect fluorescence lifetime values. Therefore, fluorescence decay is a good experimental tool for studies of protein dynamics, including thermal fluctuations and protein-ligand association.

\subsection{Excitation transport}

It is well known that in proteins excitation transport occurs through variety of paths (channels), e.g. electron transfer (and/or hole transfer) or fluorescence resonance energy transfer (FRET) from excited tryptophan and/or tyrosine residues. Since no indication for energy acceptors for FRET in proteins have been found [23], we consider fluorescence decay modeling which involves electron transport processes. The general idea is that the electron of an excited fluorophore can migrate (with a transfer rate $\nu(t)$ ) to some excited states different from the initial one. One example of such a process in proteins is electron transport to a neighboring quenching residue. Many possible electron acceptors have been proposed, such as a peptide bond carbonyl group [23] (due to appearance of a net positive charge on the peptide bond carbonyl carbon [24]) or amino acid residues involved in $\pi-\pi$ stacking interaction. Since almost all amino acid side chains are more or less effective quenchers $[10,25]$, the proposed model is applicable to proteins in general.

We start with the rate equation for excitation transport (with natural fluorescence lifetime $\tau_{0}$ ), across a medium containing a relative concentration $c$ of electron acceptors, given by the rate equation [26]

$$
\frac{\mathrm{d} I(t)}{\mathrm{d} t}=-c \nu(t) I(t)-\frac{1}{\tau_{0}} I(t) .
$$

The stochastic character of non-stationary excitation transport can be accounted for by the time-dependence of the transfer rate [5]

$$
\nu_{i j}(t)= \begin{cases}\nu_{0} \exp \left(-\varepsilon_{i j} / k T\right)\left[(1-\omega)+\omega \exp \left(-\varepsilon_{i j} t / \Gamma\right)\right], & \varepsilon_{i j}>0 \\ \nu_{0}, & \varepsilon_{i j} \leq 0\end{cases}
$$

where $\varepsilon_{i j}=E_{i}-E_{j}$ ( $E_{i}$ is the energy of site $i$ ), $\Gamma$ is a factor associated with the density of the excited states, and the coefficient $\omega$ describes the noise filtration in diffusion processes.

This detailed balance implies that the forward rates connecting a pair of sites are related by the Boltzmann factor. The exponential relaxation can be explained in terms of a random walk, i.e. in terms of the number of distinct sites, $S(t)$, visited by a random walker in equivalent media, since the transfer rate is related to $S(t)$ by the relation $\nu(t) \propto \mathrm{d} S(t) / \mathrm{d} t$. In particular the white noise with low frequency filtration implies that relaxation starts from $S(t=0)=0$ and tends asymptotically to $S(t) \propto t$ behavior (Fig. 1). Consequently, $\nu(t)$ tends to a constant value and leads to a non-stationary process with exponential re- 


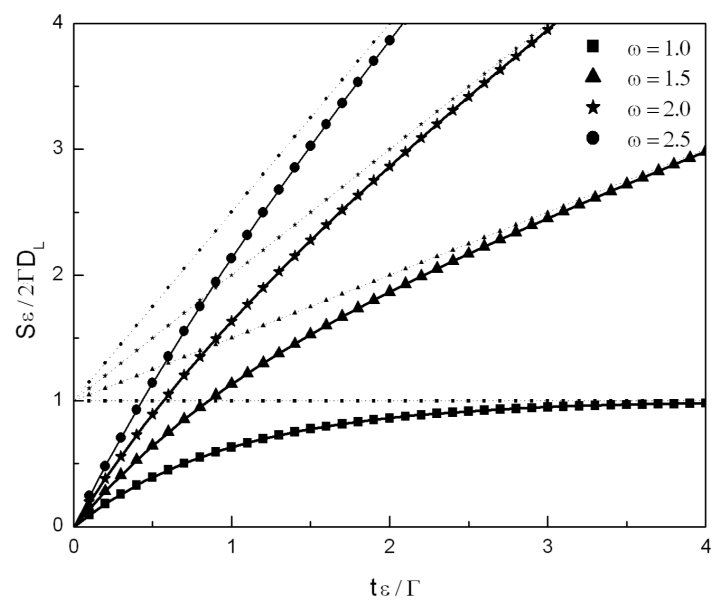

Fig. 1. Time dependence of $S(t)$ for the white noise with low frequency filtration defined by $S(t)=2\left(D-D_{\mathrm{L}}\right) t+2 D_{\mathrm{L}} \Gamma / \varepsilon-\left(2 D_{\mathrm{L}} \Gamma / \varepsilon\right) \exp \left(-\frac{\varepsilon}{\Gamma} t\right)$, and $S \varepsilon /\left(2 \Gamma D_{\mathrm{L}}\right)$ plotted as a function of $t \varepsilon / \Gamma$ for different values of coefficient $\omega=D / D_{\mathrm{L}}$ (see Ref. [5] for details).

laxation [5]. This, in turn, implies that, when relaxation takes place, the higher energy sites become increasingly difficult to attain, so that the number of accessible sites decreases with time [27].

The energy averaged transfer rate can be formally derived from integration of Eq. (9) over energies and is equal to

$$
\langle\nu(t)\rangle=\nu_{0}(1-\omega)+\frac{\nu_{0} \omega \Gamma}{\Gamma+k T t} .
$$

Taking into account the above transfer rate, the rate equation for excitation (Eq. (8)) leads to

$$
I(t)=I_{0} \exp \left(-\frac{t}{\tau_{1}}\right)\left(1+\frac{1}{\gamma} \frac{t}{\tau_{2}}\right)^{-\gamma},
$$

where in our case

$$
\tau_{1}=\frac{1}{1 / \tau_{0}+c \nu_{0}(1-\omega)}, \tau_{2}=\frac{1}{c \nu_{0} \omega}, \gamma=\frac{1}{q-1}, \text { and } q=1+\frac{k T}{\Gamma} \tau_{2} .
$$

The power-like term in the above distribution can be treated as a yet another example of the Tsallis $q$-exponential function. Note that the power-like term in Eq. (11) can be accounted for time hierarchy in the considered process. Considering, for simplicity, that the excited states $E_{i}=\mathrm{i} \varepsilon$ are to be reached in the $i$-th step of the hierarchy level, the transfer rate is given by $\exp \left(-E_{i} / k T\right)=\beta^{i}$, where $\beta=\exp (-\varepsilon / k T)$. Moreover, the states are usually distributed exponentially [25] (as $\exp \left(-E_{i} / k T_{0}\right)=\eta^{i}$, where $T_{0}$ describes state density). Thus the state density is given by $\eta=\exp \left(-\varepsilon / k T_{0}\right)$. According to Blumen et al. [28], taking into account excitation from all steps, 


$$
I(t)=\frac{1-\eta}{\eta} \sum_{i=1}^{\infty} \eta^{i} \beta^{i} \exp \left(-t \beta^{i}\right)
$$

Asymptotically, for the long time this lead to

$$
I(t) \sim t^{-\gamma},
$$

where

$$
\gamma=1+\frac{\ln \eta}{\ln \beta}=1+\frac{T}{T_{0}} .
$$

\section{Experimental}

\subsection{Materials}

N-acetyl-L-tyrosinamide (NATyrA) was purchased from Aldrich Chemical Company, Inc. (Mileaukee, WI, USA), and used without further purification. Formycin A (FA), mono- and disodium phosphate, and N-2-hydroxyethylpiperazine-N'-2-ethanesulfonic acid (Hepes) were products of Sigma Chemical Co. (St. Louis, MO, USA). The latter was used as a buffering medium at $50 \mathrm{mM}$ concentration, with $\mathrm{pH}$ value adjusted to 7 by addition of concentrated sodium hydroxide $(\mathrm{NaOH})$ from Merck (Darmstadt, GFR). Purine nucleoside phosphorylase (PNP; purine nucleoside: orthophosphate ribosyltransferase, EC 2.4.2.1) from E. coli, a product of the deoD gene, was prepared as described earlier [29].

\subsection{Time-resolved fluorescence measurements}

Fluorescence intensity decays were obtained by time-correlated single photon-counting (TCSPC) measurements of fluorescence, performed on an IBH time-resolved spectrofluorimeter (IBH Consultants, Scotland, UK) (see for further details Ref. [19]) at $25^{\circ} \mathrm{C}$ in the presence of natural $(0.25 \mathrm{mM})$ concentration of oxygen. The latter is too low to affect tyrosine fluorescence intensity decay, as was previously shown by measurements of bimolecular oxygen-quenching constants and fluorescence lifetimes for L-tyrosine, NATyrA, and tyrosine-containing peptides and proteins [30]. Typical fluorescence intensity decay $(I)$ data were convoluted with the instrument response and fitted to models using interactive convolution. The quality of fit was evaluated by the structure observed in the plots of residuals normalized to error, i.e. residual $=\frac{I_{\text {experimental }}-I_{\text {theoretical }}}{\sqrt{I_{\text {experimental }}+I_{\text {theoretical }}}}$, and by the reduced chi-square value.

\section{Results}

The power-like decay function provides a good fit to complex fluorescence decays by linking the character of the decay law to the distribution of lifetimes [19]. The acetyl-amide form of L-Tyr (NATyrA), which exists exclusively in the neutral 
form in aqueous solution at neutral $\mathrm{pH}$, is a good model compound for phenolic residues in a polypeptide chain. NATyrA exhibits a complex fluorescence decay usually not described by a single exponential term [8,9]. Figure 2 shows the fluorescence decay for NATyrA in aqueous solution. Bad fits were obtained for the single as well as double exponential model, as judged by residuals $\left(\chi_{\mathrm{R}}^{2}=4.93\right.$ and 1.85 , respectively) [19]. In contrast, a very good fit $\left(\chi_{\mathrm{R}}^{2}=1.20\right)$ was obtained with the power-like decay function, and $q=1.07 \pm 0.02$, which leads to the decay path value $N \approx 285$, and may reflect free fluorophore in solution. Although applicability of the multi-exponential models are not physically justified, the resultant mean decay time $(\langle t\rangle=1.63 \pm 0.05 \mathrm{~ns})$ for NATyrA fluorescence is in agreement with that obtained with the power-like model $(\langle t\rangle=1.53 \pm 0.08 \mathrm{~ns})$.

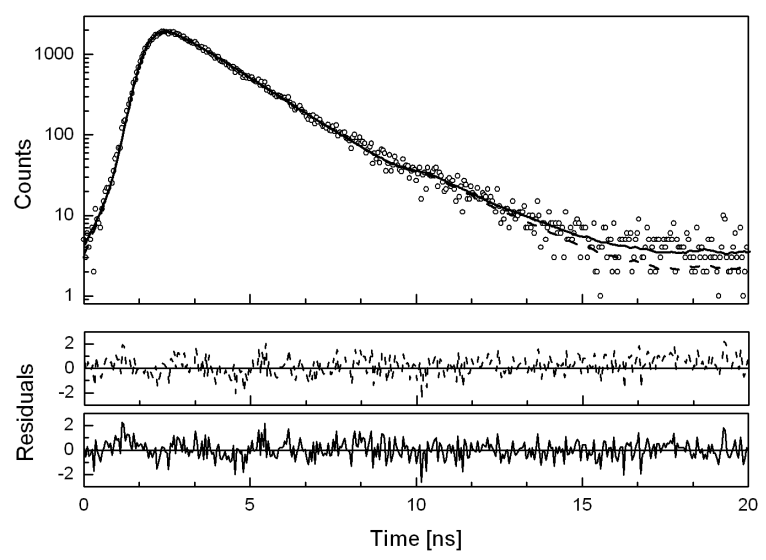

Fig. 2. Fluorescence intensity decay $\left(\lambda_{\text {exc }} / \lambda_{\text {em }} 275 / 320 \mathrm{~nm}\right)$ of NATyrA shown by circle. The solid and dashed curves represent the theoretical values of the best fits of Eq. (5) (with fitted parameters $q=1.07 \pm 0.02, \tau_{0}=1.32 \pm 0.03 \mathrm{~ns}$ and $\langle t\rangle=$ $1.53 \pm 0.08 \mathrm{~ns}$ ), and single-exponential function (with $\tau=1.63 \pm 0.01 \mathrm{~ns}$ ), respectively.

E. coli purine nucleoside phosphorylase (PNP) and its ternary complex with FA (inhibitor) and phosphate (natural co-substrate) in aqueous solutions was chosen as a good examples of highly complex fluorescence intensity decays resulting from excitation of tyrosine residues in the enzyme, the $\mathrm{N}(1)-\mathrm{H}$ and $\mathrm{N}(2)-\mathrm{H}$ tautomeric forms of FA, free in solution (see Ref. [31] and literature cited therein), and the latter bound in the active site of the enzyme [29, 31]. Fluorescence decay of such a complex system cannot be properly described using a single-exponential model [29], due to many interacting fluorophore residues, which prevent consideration of the individual decay times [16]. In addition, the kinetics of fluorescence decay is affected by FRET, i.e. the resonance interaction by weak (Förster) coupling between transition dipoles of emission and absorption of protein tyrosine residues (Tyr160) and the $\mathrm{N}(2)-\mathrm{H}$ form of FA bound by the enzyme [32]. Moreover, crystal data and previous investigations (see Ref. [32] and literature cited 
therein) indicate that both the base moiety of FA and the phenol ring of Tyr160 are involved in $\pi-\pi$ interactions with the aromatic residue of Phe159. The latter is located between FA and Tyr160, almost perpendicularly to the base moiety of FA from one side and to the phenol ring of Tyr160 on the other side. Such $\pi-\pi$ stacking implies involvement of higher order multipole couplings, as well as exchange interactions. Therefore, a model involving the excitation transport process seems to be more appropriate than a one considering a sum of the individual decay components. Indeed, we observed that the decay function given by Eq. (11) provides a good fit of the fluorescence decay data of both the PNP enzyme free in solution (Fig. 3) and the PNP-FA-P $i$ complex (Fig. 4) with $\chi_{\mathrm{R}}^{2}=1.02$ and 1.1 , respectively, and a high accuracy of the fitted parameters (see captions in Figs. 3 and 4). By contrast, poor fits were obtained with the single-exponential model $\left(\chi_{\mathrm{R}}^{2}=6.28\right.$ and 2.64), somewhat improved with the double-exponential model $\left(\chi_{\mathrm{R}}^{2}=1.02\right.$ and 1.32), but the residuals (Figs. 3 and 4$)$ confirm that the decay function (Eq. (11)) is still better than the double-exponential function [29]. Furthermore, the increasing number of exponential terms in the multi-exponential model is justified only by the resultant improvement of the goodness of fit, without physical interpretation of the decay components.

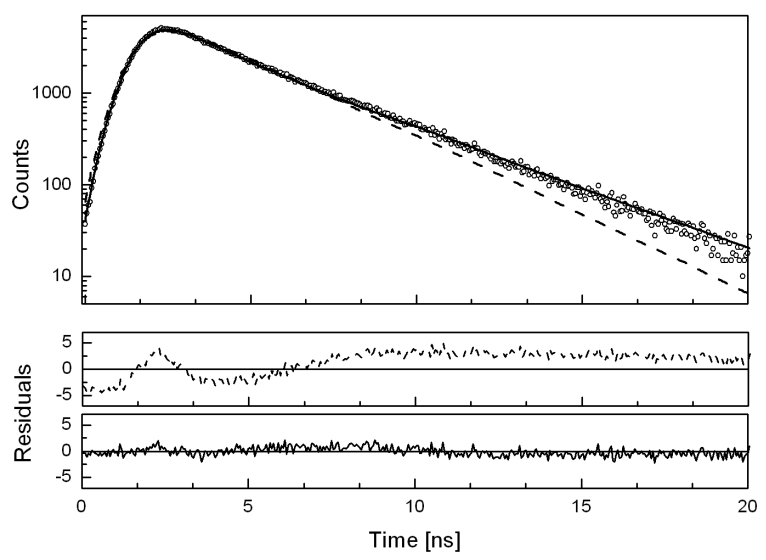

Fig. 3. Fluorescence intensity decay $\left(\lambda_{\mathrm{exc}} / \lambda_{\mathrm{em}} 275 / 310 \mathrm{~nm}\right.$ ) of $E$. coli PNP (free in aqueous solution) shown by circles. The solid and dashed curves represent the theoretical values of the best fits of Eq. (11) (with fitted parameters $\tau_{1}=3.51 \mathrm{~ns}, \tau_{2}=0.1 \mathrm{~ns}$ and $\gamma=0.33$ ), and single-exponential function (with $\tau=2.3 \mathrm{~ns}$ ), respectively.

These results show that, due to the complex formation, the natural fluorescence lifetime of the enzyme-ligand complex is shorter than that of the free protein. In contrast, the lifetime $\tau_{2}$ associated with electron transfer phenomena did not change significantly, which further supports hypotheses that the excitation transfer occurs between residues involved in the $\pi-\pi$ stacking. 


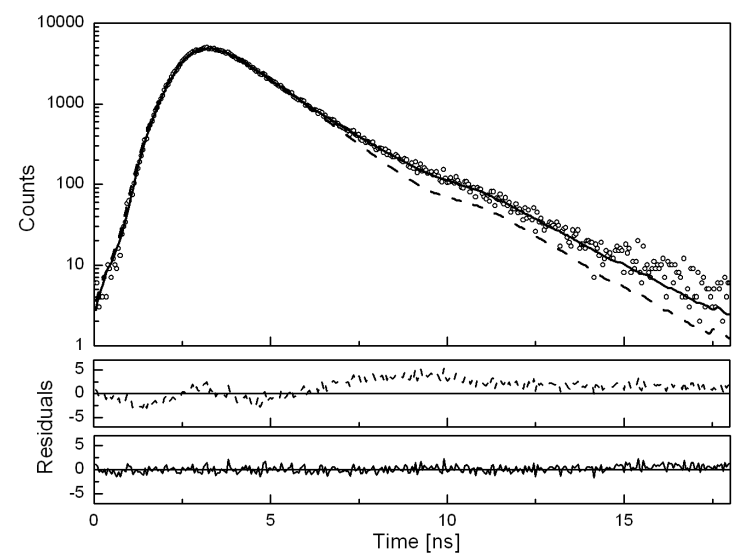

Fig. 4. Fluorescence intensity decay $\left(\lambda_{\text {exc }} / \lambda_{\mathrm{em}} 275 / 310 \mathrm{~nm}\right)$ of $E$. coli PNP in its ternary complex with formycin A and phosphate shown by circles. The solid and dashed curves represent the theoretical values of the best fits of Eq. (11) (with fitted parameters $\tau_{1}=1.88 \mathrm{~ns}, \tau_{2}=0.13 \mathrm{~ns}$ and $\gamma=0.44$ ), and single-exponential function (with $\tau=$ $1.46 \mathrm{~ns})$, respectively.

It is worth noting that the decay of fluorescence of the PNP ternary complex may be also fitted by a power-like function (Eq. (5)) with $\langle t\rangle=1.49 \pm 0.04 \mathrm{~ns}$ and $q=1.105 \pm 0.001$, which leads to a decay path value $N \approx 19$. This is in contrast to the fluorescence decay of the PNP enzyme free in aqueous solution (data not shown), described by a power-like function with $\langle t\rangle=3.13 \pm 0.05 \mathrm{~ns}$ and $q=1.25 \pm 0.02$, i.e. with $N \approx 8$, reflecting the fact that the complex formation leads to an increase number of the relaxation paths associated with enzyme-ligand interactions.

\section{Conclusions}

We show here that the application of continuous lifetime distributions for interpretation of complex fluorescence decays are more valid than multi-exponential models, where the interpretation of individual components is incorrect, and the definition of parameters is unclear. A good alternative to a multi-exponential function may be the power-like decay function obtained directly from statistical treatment of the fluorescence decays based on the gamma-distributed fluctuations of the fluorescence lifetime. When the heterogeneity parameter $q \rightarrow 1$ (the number of decay paths $N \rightarrow \infty$ ), i.e. lifetime distribution becomes a $\delta$ function, a powerlike function leads to a mono-exponential function describing an ideal decay [19].

Since the power-like function (Eq. (5)) is determined by only two parameters (the mean value of lifetime distribution and relative variance), it is advantageous for multi-exponential models by increased efficiency of fitting of the complex fluorescence decays. The importance of the latter has been verified for a wide range 
of cases [19], and suggests that its application in on-line analysis of the fluorescence lifetime imaging (FLIM) data should significantly speed up refinement of the lifetime images and improve their contrast and quality, similarly to that observed with stretched exponential function [33].

As it was already stated, one of the possible origin of fluorescence decay heterogeneity are electron transfer processes. In that case fluorescence intensity decays may be described by an equation containing an exponential function multiplied by a power-like term (Eq. (11)) being yet another example of the Tsallis $q$-exponential function. The latter can be accounted for time hierarchy in the considered processes, and relates the power index $\gamma$ with density of excited states.

\section{Acknowledgment}

This research was supported by the State Committee for Scientific Research (KBN), which includes grants 3P04A02425, BW-1605/BF and BST-975/BF.

\section{References}

[1] C. Tsallis, Braz. J. Phys. 29, 1 (1999).

[2] C. Tsallis, Phys. World 10, 42 (1997).

[3] C. Tsallis, J. Stat. Phys. 52, 479 (1988) For updated bibliography on this subject see http://tsallis.cat.cbpf.br/biblio.htm cf. also Special issue of Braz. J. Phys. 29, (1999) available at http://sbf.if.usp.br/bjp/Vol29/Num1/index.htm.

[4] H. Frauenfelder, P.G. Wolynes, R.H. Austin, Rev. Mod. Phys. 71, S419 (1999).

[5] J. Wlodarczyk, B. Kierdaszuk, Chem. Phys. 297, 139 (2004).

[6] J.R. Lakowicz, Photochem. Photobiol. 72, 421 (2000).

[7] J.B.A. Ross, H.R. Wyssbrod, R.A. Porter, G.P. Schwartz, C.A. Michaels, W.R. Laws, Biochem. 31, 1585 (1992).

[8] J.B.A. Ross, W.R. Laws, K.W. Rousslang, H.R. Wyssbrod, in: Topics in Fluorescence Spectroscopy, Ed. J.R. Lakowicz, Vol. 3, Plenum Press, New York 1992, p. 1.

[9] J.B.A. Ross, W.R. Laws, A. Buku, J.C. Sutherland, H.R. Wyssbrod, Biochemistry 25, 599 (1986).

[10] B.S. Hudson, J.M. Huston, G. Soto-Campos, J. Phys. Chem. 103, 2227 (1999).

[11] J.R. Alcala, E. Gratton, F.G. Prendergast, Biophys. J. 51, 925 (1987).

[12] A.G. Szabo, T.M. Stepanik, D.M. Wayner, N.M. Young, Biophys. J. 41, 233 (1983).

[13] I. Gryczynski, W. Wiczk, M.L. Johnson, J.R. Lakowicz, Biophys. Chem. 32, 173 (1988).

[14] A.S. Ladokhin, S.H. White, Biophys. J. 81, 1825 (2001).

[15] P.R. Callis, Methods Enzymol. 278, 113 (1997).

[16] J.R. Lakowicz, Principles of Fluorescence Spectroscopy, 2nd ed., Kluwer Academic/Plenum Publisher, New York 1999, p. 130. 
[17] M.L. Mehta, Random Matrices, Academic Press, New York 1991.

[18] G. Wilk, Z. Włodarczyk, Phys. Lett. A 290, 55 (2001).

[19] J. Włodarczyk, B. Kierdaszuk, Biophys. J. 85, 589 (2003).

[20] G. Wilk, Z. Włodarczyk, Phys. Rev. Lett. 84, 2770 (2000).

[21] G. Careri, P. Fasella, E. Gratton, Ann. Rev. Biophys. Bioeng. 8, 69 (1979).

[22] M. Karplus, J.A. McCammon, Ann. Rev. Biochem. 53, 263 (1983).

[23] A. Ababou, E. Bombarda, Protein Sci. 10, 2102 (2001).

[24] E.J. Milner-White, Protein Sci. 6, 2477 (1997).

[25] Y. Chen, M.D. Barkley, Biochemistry 37, 9976 (1998).

[26] R. Richert, H.J. Bässler, Chem. Phys. 84, 3567 (1986).

[27] R.P. Parson, R. Kopelman, J. Chem. Phys. 82, 3692 (1985).

[28] A. Blumen, J. Klafter, G. Zumofen, in: Fractals in Physics, Eds. L. Pietronero, E. Tosatti, Elsevier, Amsterdam 1986, p. 399.

[29] B. Kierdaszuk, A. Modrak-Wójcik, J. Wierzchowski, D. Shugar, Biochim. Biophys. Acta 1476, 109 (2000).

[30] J.R. Lakowicz, B.P. Maliwal, J. Biol. Chem. 258, 4794 (1983).

[31] B. Kierdaszuk, in: Fluorescence Spectroscopy, Imaging and Probes. New Tools in Chemical, Physical and Life Sciences, Eds. R. Kraayenhof, A.J.W.G. Visser, H.C. Gerritsen, Vol. 2, Springer-Verlag, Heidelberg 2000, p. 277.

[32] J. Wlodarczyk, G. Stoychev-Galitonov, B. Kierdaszuk, Eur. Biophys. J. 33, 377 (2004).

[33] K.C. Benny Lee, J. Siegel, S.E.D. Webb, S. Leveque, M.J. Cole, R. Jones, K. Dowling, M.J. Lever, P.M.W. French, Biophys. J. 81, 1265 (2001). 